1

2

4

\title{
Effect of host-switching on the eco-evolutionary patterns of parasites
}

${ }^{1 *}$ Elvira D’Bastiani, ${ }^{2}$ Débora Princepe,${ }^{1,3}$ Karla M Campião, ${ }^{2}$ Flavia MD Marquitti,,${ }^{1,3}$ Walter A Boeger,

${ }^{1,4}$ Sabrina LB Araujo

${ }^{1,3,4}$ Laboratório de Interações Biológicas, Universidade Federal do Paraná, UFPR

${ }^{3}$ Departamento de Zoologia, Universidade Federal do Paraná, UFPR

${ }^{2}$ Departamento de Física, Universidade Estadual de Campinas, UNICAMP

${ }^{4}$ Departamento de Física, Universidade Federal do Paraná, UFPR

*Corresponding author: Elvira D’Bastiani e-mail: elviradbastiani@gmail.com

\section{Authorship statement:}

Conceived and designed the experiments: EDB, SBLA and DP

Performed the experiments and analysed the data: EDB, SBLA and DP

Wrote the paper: EDB, SLB, KMC, DP, WB, FMDM

Other contributions: EDB, DP, FMDM, WB, KMC and SLBA

Emails:

Elvira D’Bastiani <elviradbastiani@gmail.com>

Débora Princepe <deborapr@gmail.com>

Karla M. Campião <kcampiao@gmail.com>

Flavia MD Marquitti<flamarquitti@gmail.com>

Walter A Boeger <wboeger@gmail.com>

Sabrina LB Araujo <araujosbl@gmail.com> 


\section{ABSTRACT}

31 Increasing empirical evidence has revealed that host-switching is more common than cospeciation

32 in the history of parasites. Here, we investigated how the intensity of host-switching, mediated by

33 opportunity and compatibility, affects the phylogenetic history and ecology of the parasites. We

34 developed a theoretical model to simulate the evolution of populations of parasites that can

35 explore and colonize new hosts under variable host-switching intensities. Eco-evolutionary

36 patterns (beta diversity/normalized Sackin index) obtained from parasite simulations were

37 compared to nine empirical cases. Our model reproduced the empirical patterns, and such

38 simulations varied in host-switching intensity according to the analysed case. This intensity does

39 not differ among cases of ecto and endoparasites, but it was stronger in local cases when compared

40 to a regional scale. Our results highlight the importance of contact opportunity, and suggest that

41 host-switching intensity mediates the exploration and colonization of new hosts promoting

42 variation in the eco-evolutionary patterns.

43 Key-words: dispersion of parasites, opportunity and compatibility of interaction, phylogenetic

44 conservatism and structure of community of parasites.

45 


\section{INTRODUCTION}

The dispersion of parasite individuals followed by colonization of a new host lineage, known as host-switching, is a common event observed during the evolutionary trajectory of many lineages of parasites (De Vienne et al. 2013). Initially, host-switching results in the increase of the host repertoire of a parasite (Braga et al. 2021) which, subsequently, when new hosts can impose reproductive isolation it may result in speciation of parasite lineages. This is the dynamic of the oscillation hypothesis (Nylin \& Soren 2018). Empirical examples showing high levels of host-switching include symbiotic interactions ranging from host-parasite and plant-insect systems to microbial pathogens, brood parasitism, plant-feeding insects, and even parasitic plants (Nylin et al. 2014; Fecchio et al. 2019; Hayward et al. 2021). Consequently, understanding the factors influencing the success of host-switching and subsequent speciation events is critical for understanding the parasite diversification.

A recently proposed theoretical framework that accommodates the evolutionary dynamics of host-parasite associations, the Stockholm Paradigm (Brooks et al. 2014; Brooks et al. 2019), suggests that parasites perform host-switching by ecological fitting (Agosta \& Klemmens 2008; Agosta \& Brooks 2020). Ecological fitting is the process whereby organisms colonize and persist in novel environments, use novel resources or form novel associations with other species through a set of traits they already possess (Agosta \& Klemmens 2008). The expression of these unexplored capabilities is mediated by the opportunity of interaction (temporal, spatial, ecological), which determines the possibility of encounters between hosts and unfamiliar parasites. After the encounter, and if the interaction is compatible, it is followed by the resolution of subsequent conflicts that emerge from the basic dynamics of "living together", which should result in coaccommodation (Brooks \& McLennan 2012; Araujo et al. 2015). 

influenced also by ecological and life-history traits. These include characteristics of all organisms within the interaction system, such as niche similarity among host species, modes of transmission of parasites, dietary preferences of the vector (if there is one), and also ecosystemic characteristics as the host community composition and shared phylogenetic history are relevant factors that define the chances of host-switching (Bush et al. 2006; Jaramillo \& Rivera-Parra 2018). Niche similarity among host species is one fundamental element constraining the incorporation of new host species by ecological fitting. This is because the capacity of a parasite species to use new resources is related to the phylogenetic conservatism of the resource provided by the host species. Phylogenetic distance between the original and new host species represents an adequate proxy for the nature of the resource which is tracked by the parasite lineage (Charleston \& Robertson 2002; Agosta \& Klemmens 2008; Engelstädter \& Fortuna 2019). Consequently, the host phylogenetic conservatism can define the arena of possibilities for host-switching.

Several studies have indicated the ubiquity and relevance of host-switching in nature (Engelstädter \& Hurst 2006; De Vienne et al. 2007; Cuthill \& Charleston 2013; De Vienne et al. 2013; Engelstädter \& Fortuna 2019). Although, few have explored the relationship between the switches and the evolutionary histories of host species (see

101 Cuthill \& Charleston 2013; Engelstädter \& Fortuna 2019). Moreover, the effect of 102 compatibility and interaction opportunity for parasite propagation between host species, 103 as well as the influence of such factors in the patterns of parasite communities remain 104 unexplored. Here, we aim to fill these unexplored gaps by proposing a novel approach to investigate how the intensity of host-switching, mediated by opportunity and 106 compatibility of interaction, affects the phylogenetic history and ecology of the parasites. 
107 We proposed a theoretical model based on parasite individuals that can switch among

108 host species during their evolution according to phylogenetic conservatism; that is, the

109 probability of parasites switching hosts decreases with increasing divergence in the

110 evolutionary time of the host. We compared the eco-evolutionary pattern that emerged

111 from the model to nine empirical communities of parasites to predict their intensity of

112 host-switching. These communities were classified by the parasitism type (ecto or

113 endoparasites) and their spatial scale (local or regional). We analysed how the predicted

114 host-switching varied over these classifications and discussed how they are mediated by

115 opportunity and compatibility of interaction.

117 MATERIAL AND METHODS

118 Model general overview

119 We modeled the eco-evolutionary trajectories of parasites influenced by their host

120 evolutionary history and host-switching events. Thus, we assumed that the evolutionary

121 history of the host can represent a proxy for the resources for parasite species (Agosta et

122 al. 2010; Imrie et al. 2021). We also assumed that the probability of host-switching

123 decreases as the phylogenetic distance between the species of host involved in the event

124 (original and new host species) increases. Finally, the model assumes that parasite

125 evolution occurs at the same evolutionary time scale as the host, which increases

126 possibilities for host-switching as host speciation occurs. Parasite individuals are

127 explicitly modeled and characterized by their used host species and genetic identity,

128 following the description of the model proposed by Higgs \& Derrida (1991) and Manzo

$129 \&$ Peliti (1994). This approach allows us to model parasite speciation. The host species

130 are modeled as resources that impose a carrying capacity to parasite species, analogous

131 to islands in the Manzo-Pelit model (Manzo \& Peliti 1994), but in the present model, the 
132 islands (hosts species in our case) emerge (as a new host species that speciate) and the

133 distance between them varies over time, according to a predetermined time host

134 diversification (e.g. based on ultrametric empirical phylogenies). The model does not

135 consider the selection pressure imposed by parasites on the evolution of the resource.

136 Therefore, we are not modeling a process of reciprocal evolution, or co-evolution.

138 Host and parasite characterization

139 Following the model proposed by Higgs \& Derrida (1991), parasite individuals

140 are explicitly modeled by biallelic sequences of infinite sites, a simplified form to

141 represent their genomes and heritable trait. Individuals are monoic and engage in sexual

142 reproduction, with non-overlapping generations. The empirical data have evolutionary

143 times in the order of millions of years, and to maintain this time scale in the model would

144 demand a higher computational cost. As proposed by Costa et al. (2019), in our approach

145 we adopted a higher value of mutation rate in order to decrease the number of iterations

146 necessary for speciation (time steps or generations) to happen. Furthermore, we assumed

147 that, due to the shorter life cycle of parasites, they have a faster speciation rate (Dowton

148 \& Austin 1995; Light \& Hafner 2007). To satisfy these conditions, we rescaled the whole

149 host phylogeny assuming that the smaller branch length consists of the minimal time for

150 parasites to speciate in allopatry ( $\boldsymbol{\tau}_{a}$ generations - see the details in supplemental

151 information I). The simulation starts with a clonal parasite population using a unique host

152 species. The first host speciation occurs only after $\boldsymbol{\tau}_{a}$ generations, accumulating genetic

153 variation before the first host speciation (the root of the host phylogeny). Each host

154 species imposes a carrying capacity of $K$ parasite individuals. Thus, the overall carrying

155 capacity increases by $K$ individuals at each new host speciation. 


\section{Parasite reproduction}

Reproduction is sexual and occurs between parasite individuals that are in the same host and that have a minimum genetic similarity, $\mathrm{q}_{\min }$, measured based on the

160 Hamming distance between genomes. In each host species, $K$ offspring are born,

161 replacing the parental population. We establish a maximum of $K$ random trials with

162 reposition to find one compatible partner. The offspring is generated by locus 163 recombination of the parents and each locus has a probability $\mu$ of mutation. We set $164 q_{\min }=0.5 q_{0}$, where $q_{0}$ is the expected mean similarity within one population in 165 equilibrium, because it avoids the occurrence of parasite speciation within the same host

166 species. Consequently, parasite speciation only happens when more than one host species

167 is used. For a more detailed description of the method, see the supplemental information 168 I.

\section{Host-switching}

After the first host speciation, parasite individuals in a host species can switch to another host. For each parasite individual, we randomly selected a host species, including the one in use. If the selected host species is not the original host (donor), we follow a probability function for the host-switching event. This probability of host-switching events $\left(P_{h s}\right)$ decreases over time, representing the loss of opportunity and/or compatibility of parasites associated with the evolutionary history of hosts. The probability of a parasite

177 individual successfully migrate (host-switching) from one host to another host species, in a given generation $n$, is defined as:

$$
P_{h s}(n)=\exp \left[-r *\left(n-n_{s}\right)\right],
$$

where $r$ is a positive parameter that controls the decay of the host-switching probability, 
183 long the two host species had diverged). If $r=0$, these probabilities are equal to 1

184 regardless of the host divergence time, meaning that there is no restriction to host-

185 switching. As a consequence, parasite gene flow is continuous and speciation does not

186 occur. At the other extreme, for sufficiently large $r$ values $\left(\mathrm{P}_{h s} \sim 0\right)$, host-switching is

187 absent, and cospeciation between hosts and parasites is expected. For intermediary $r$

188 value, some parasite individuals can eventually switch hosts. This will increase the host

189 repertoire of the parasitic species, and also enable speciation by isolation (by host use),

190 similar to the speciation by founder's effect (Mayr 1999; Gavrilets \& Hastings 1996). In

191 order to better interpret the effect of parameter $r$ on the trajectories and compare the

192 results between the communities, we do not present our results in terms of $r$, but how

193 much it changes the overall host-switching events. To obtain this overall metric, we

194 calculated the expected percentage of parasite individuals that switch hosts over the entire

195 simulation and we call it as host-switching intensity.

\section{Empirical database}

Nine studies from empirical communities of parasite-host associations (Table 1)

199 were used for comparative purposes. Selection criteria was that, in addition to information

200 on species interaction, these communities essentially needed to have published

201 phylogenies for hosts and parasites (see the details in supplemental information II). We

202 separated these communities according to the parasitism type and spatial scale (Table 1 -

203 Fig. S5-S13). For all empirical studies selected, parasite groups are monoxenic (both ecto-

204 endoparasites), with simple life cycles that encompasses a single host species. All

205 ectoparasites analysed herein can be transmitted during the larval and adult stages while

206 endoparasites can be transmitted only during the larval stage. Hence, ectoparasites are

207 likely more prone to explore and colonize new hosts (Boeger et al. 2005; Malcicka et al. 
2015). A total of 50 runs were performed with 250 individuals of carrying capacity, for each configuration of the parameters of host-switching intensity.

\section{Characterization of the ecological and evolutionary patterns of parasites}

We expected that the ecological and evolutionary processes leave signatures in the resulting composition of parasite species and phylogenetic trees of parasites; thus, we compared both the structure of phylogenies and the composition of the parasite species in the empirical data with those resulting from the simulations. We compared the species composition using the beta diversity of multiple-site dissimilarities ( $\beta$ - Baselga 2010; 2013a, b). The structure of the phylogenetic trees was characterized by measuring the tree imbalance, using the normalized Sackin index ( $\mathrm{I}_{n}$ - Blum \& François 2005). As each empirical case represents particular ecological and evolutionary processes, we analysed whether there was an optimal range of host-switching intensity that reproduced each composition $(\beta)$ and normalized Sackin index $\left(I_{n}\right)$. To reproduce the scenarios that best fit the empirical situations, we assumed that the simulations needed to reproduce both the $\beta$ and the $I_{n}$ metrics simultaneously (with a $\pm 5 \%$ confidence interval). Then we compared the resulting host-switching intensity among the empirical cases analysed to understand how it varied for different evolutionary histories. Statistical analyses were performed using 'ape' (Paradis \& Schliep 2019), 'betapart' (Baselga et al. 2018) 'picante' (Kembel et al. 2010), ‘phytools' (Revell 2012) and ‘vegan' (Oksanen et al. 2013) R packages.

\section{Statistical analysis}

To test whether the parasitism type and spatial scale modulates the host-switching

231 intensity in the empirical cases analysed, a linear mixed-effects model (LMM) was

232 performed using the lmer function from the 'lme 4' package (Bates et al. 2015). For this 
234 fixed variables were included, and empirical cases were treated as random variables. After

235 performing the LMM analysis, an analysis of variance (ANOVA) was used to determine

236 significant differences ( $\mathrm{p}$-value $\leq 0.01)$ using the Anova function in the 'car' package

237 (Fox \& Weisberg 2019). All statistical analyses were performed in R v.4.0.0 (R Core

238 Team 2020) and Rstudio v.1.3.959 (RStudio Team 2020).

\section{RESULTS}

The beta diversity and normalized Sackin index of parasites varied according to

242 the host-switching intensity (e.g. the expected percentage of parasite individuals that

243 switch hosts during the entire history of the host community). To illustrate the prevalent

244 beta diversity and normalized Sackin index according to the host-switching intensity, we

245 presented the case of feather mites associated with birds (ID. 1 - Fig. 1). As expected,

246 beta diversity decreases as host-switching intensity increases regardless of the studied

247 case (Fig. 1a and Fig. S14). It occurs because the increase of host-switching promotes the

248 interaction of different host species with the same parasite species. Additionally, for each

249 value of host-switching intensity, there is a small variation in the beta diversity (Fig. 1a

250 and Fig. S14). The only exception was ID. 4, which resulted in a wide variation in beta

251 diversity under high host-switching intensity (Fig. S14).

$\left(\mathrm{I}_{\mathrm{n}}\right)$ for the simulated parasite phylogenies assumes exactly the same value of the one

254 obtained from the empirical phylogeny of the host (note the dashed line in Fig. 1b and

255 also Fig. S15). This low host-switching intensity does not allow the establishment of a

256 population in a new host. As a consequence, the simulated parasite phylogenies have the

257 same normalized Sackin index of the empirical host phylogeny. For higher host-switching

258 intensity, colonization followed by speciation is more likely to occur and the normalized 
259 Sackin index varies over simulations even when they are under the same host-switching

260 intensity (Fig. 1b and Fig. S15). The wide variation in the normalized Sackin index for a

261 given host-switching intensity reveals that stochastic host-switching events, even if more

262 likely occurring between closely related species, can change the structure of the resulting

263 phylogenetic tree. The normalized Sackin index tends towards zero (balanced tree) as

264 host-switching intensity goes to one, regardless of the community (Fig. S15), resembling

265 a neutral speciation scenario Yule model (Yule 1924; Aldous 2001).

For all cases, there is a host-switching intensity that simultaneously reproduces

267 the empirical beta diversity and the parasite normalized Sackin index (Fig. 2). As

268 mentioned before, both metrics are sensitive to host-switching intensity, but still restricted

269 to some combinations of beta diversity and Sackin index (see in Fig. 2, the graphs

270 showing the beta diversity vs. normalized Sackin index are not fully filled). Generally,

271 the beta diversity and the parasite normalized Sackin index obtained under high host-

272 switching intensity (greater than 50\%) are far from the empirical pattern (see Fig. 2, the

273 yellow dots rarely approach the intersection of the solid lines). Then, host phylogeny is

274 an important proxy for host-switching events.

275 The simulated host-switching intensity that simultaneously fit beta diversity and

276 parasite normalized Sackin index of each empirical case $( \pm 5 \%$ confidence interval)

277 recovered a range of $0.06 \%$ to $22.07 \%$ of host-switching intensity. Within this range, the

278 associations between mammals and lice presented the lowest host-switching intensity

279 (case ID. 2 with $0.07 \%-1.13 \%$ ), followed by that involving birds and feather mites (case

280 ID. 1 with $0.06 \%$ - 8. 17\%), wildlife and arthropod parasites (case ID. 3 with $0.82 \%$ -

281 2.69\%), frogs and Polystomatidae (case ID. 7 with $0.22 \%-3.71 \%$ ), frogs and Rhabdias

282 spp. (case ID. 8 with $1.99 \%$ - 4.94\%), frogs and Oswaldocruzia spp. (case ID. 9 with

$2835.29 \%$ - 5.91\%), fish and Gyrodactylidae (case ID. 6 with 9.35\%), - the highest intensities 
of host-switching were observed between rodents and fleas (case ID. 5 with $13.47 \%$ switching intensity and parasitism type (LMM: beta= $-0.007, \mathrm{SE}=0.018, \mathrm{df}=5.98, \mathrm{t}=-$ 0.6, $\mathrm{p}=0.70$, ANOVA: $\mathrm{F}=0.16, \mathrm{p}=0.69$, Fig. 3). The host-switching events are more frequent in studies conducted in a local scale (blue color in Fig. 3) than in regional scales (salmon color in Fig. 3) (LMM: effect of host-switching intensity on spatial scale: beta= 0.08, $\mathrm{SE}=0.18, \mathrm{df}=6.12, \mathrm{t}=4.66, \mathrm{p}=0.003$, ANOVA: $\mathrm{F}=21.75, \mathrm{p}=0.003$, Fig. 3).

\section{DISCUSSION}

In this study, we proposed a theoretical model to investigate how the intensity of host-switching, mediated by opportunity and compatibility of interaction (using host phylogenetic distance as proxy), shapes the phylogenetic history and ecology of the parasites. Our results indicated that different host-switching intensities can drive distinct ecological and evolutionary patterns of the parasite lineages. The model reproduced the eco-evolutionary pattern of all analysed empirical cases, but the range of predicted hostswitching intensity varied among them. The parasitism type did not explain this variation, but the extent of the spatial scales of the empirical cases analysed present some correlation with the predicted intensity of host-switching. Host-switching intensity was higher for local empiric cases when compared to regional scale cases.

Variation in host-switching intensity through history influences the resulting ecoevolutionary patterns of the parasites involved. When the frequency of host-switching is sufficiently high, parasites can maintain the gene flow among infrapopulations of distinct

308 hosts and speciation does not occur. On the other hand, if the gene flow is reduced, 309 reduction in host-switching favours parasite speciation (see the eco-evolutionary 
310 dynamics in the movie available in supplemental information III S16 and S17). This was

311 the general pattern observed for all simulated communities, strongly suggesting that host-

312 switching is an important driver for parasite evolution. During simulations, in line with

313 the oscillation hypothesis, species first increase their host repertoire (generalize) and then

314 speciate (specialize) (Janz \& Nylin 2008; Braga et al. 2018).

315 Our results also support that host phylogenetic relationship is a good predictor of

316 host-switching - i.e. host-switching is most likely to occur between related host species.

317 There is probably some common trait, such as a specific physiological mechanism that

318 has evolved and may be being expressed through phylogeny. The dispersion of parasites

319 followed by colonization of a new host lineage has been increasingly investigated in the

320 Stockholm Paradigm (Agosta et al. 2010; Araujo et al. 2015; Nylin et al. 2018; Brooks et

321 al. 2019). For a parasite lineage, the closer (phylogenetically) the species of the original

322 and new host, the greater the possibility that the necessary combination of elements that

323 compose the adequate resource is conserved or, is at least quali- and quantitatively

324 similar. Hence, phylogenetic proximity has been widely recognized as a potential

325 criterion to anticipate the emergence of new associations (Streicker et al. 2010; Damas et

326 al. 2020). However, this is not a universal criterion, as compatibility appears to be also

327 modulated by other biological elements (e.g. morphology, genetics, ecology) associated

328 with the opportunity of encounters between hosts and parasites. This theoretical

329 framework has provided evidence on how the host-switching mediated by compatibility

330 and opportunity of interaction influence the dynamics of parasitic interactions leading to

331 species diversification (Agosta et al. 2010; Nylin et al. 2018; Brooks et al. 2019). The

332 possibility of encounter between potential symbionts in time and space, emerges from

333 geographic distributions, ecology, and inherent biological traits of the associates.

334 Compatibility emerges from the ancestral capacity in which both must be physiologically 
compatible to establish a long-term association. Compatibility and opportunity should

336 occur simultaneously to allow the establishment of new associations. Thus, any factor

337 that influences the compatibility and/or the opportunity among hosts and parasites may

338 affect the intensity of host switching by parasites. Such factors can include biological

339 and/or spatial variations, which may explain the varied predicted intensities of host-

340 switching among analysed communities.

341 The parasitism type (ecto $v s$ endoparasites) had no effect on the predicted intensity

342 of host-switching by parasites. Ectoparasites have direct contact with the external

343 environment, while endoparasites may have free-living infective stages but spend most

344 of their life without direct contact with the environment (Bush et al. 2001). Because of

345 this, higher host-switching intensity could be expected in ecto rather than endoparasites

346 due to the amount of time under variable environmental conditions that could lead to a

347 stronger selective pressure to use a broader array of hosts. In addition, all parasites

348 analysed here are monoxenic and differ by the transmission strategy: ectoparasites can

349 transmit between host individuals during adult stages, while the endoparasites only during

350 the larval stage. Hence, the strategy of transmission of the ectoparasites allows successive

351 host-switching by an individual parasite, while endoparasites are restricted to less host-

352 switching events. This wider possibility of transmission was assumed to favor a higher

353 intensity of host-switching by ectoparasites than by endoparasites (Boeger et al. 2005).

354 Consequently, as we could not detect significant differences between the empirical

355 networks analysed herein, our results do not support these hypotheses. For instance, an

356 alternative explanation may be linked to the generalization that propagule size

357 compensates for the wider possibility of transmission. Moreover, there is great

358 heterogeneity in the characteristics of both ecto and endoparasites. Each parasitism type

359 includes a great diversity of organisms, with profound differences in their evolutionary 
history and biological characteristics (for example, by comparing species of the genus

361 fleas, lice, feather mites, helminths, platyhelminthes), which may be more influential to

362 host-switching intensity than the general site of parasitism type itself. Expanding the

363 analyses to a broader sample of empirical networks, including variations in the

364 reproductive strategies may provide important insights on this question.

Unlike parasitism type, our results indicate that host-switching intensity is higher

on local than regional spatial scale. The opportunity for interaction is increased in host

communities at a local scale, as this reduces the likelihood that geographic barriers exist,

cases at regional (ID. 4) and local spatial scales (ID. 5). Similarly, since the cases of

Rhabdias spp. and frogs (ID. 8) are defined geographically (and not by host taxa) it was

assumed that host-switching and ecological fitting were evolutionarily more important

2016; Dona et al. 2017; Patella et al. 2017).

Although the model can reconstruct eco-evolutionary patterns of empirical cases,

377 it has some limitations. First, there is no variation of the carrying capacity imposed by each host species; second, variation in abundance and spatial distribution are not explicitly considered; third, the parasites compete for the same resource (in contrast, each

380 parasite species would have a carrying capacity); and finally, selective pressure is not

381 explicitly modelled. The solutions to these limitations would make the model more realistic but also demand more computational time and increase the number of parameters to be evaluated. Thus, quantitative comparisons of predicted host-switching intensities

384 should not be done, but the qualitative implications, as already discussed. Furthermore, 
we emphasize the need for empirical phylogenetic studies, since the availability of phylogenetic data on parasites is still scarce. Phylogenetic data on parasites are extremely important to clarify the role of host-switching in the ecological and evolutionary patterns of parasite lineages. and compatibility of interaction, influence the phylogenetic history and ecology of the parasites. As suggested by the Stockholm Paradigm, parasites may already carry the ancestral capabilities to switch to new hosts, and here we showed that the host evolutionary history, when associated with opportunity for contact and interaction compatibility (opportunity + compatibility are represented by the parameter $r$ ), creates the possibility for the colonization of a new host species. Our study represents a first attempt to model and evaluate the empirical evolutionary history of hosts as a proxy for parasite resources and offers a new approach to understanding the eco-evolutionary patterns of parasite species. This model has important implications for predicting changes in host lineages in situations of environmental or climatic changes or yet in cases of emerging diseases where the parasitic host switches can cause disease outbreaks.

401

\section{ACKNOWLEDGEMENTS}

We thank the researchers who collected and reported the field data, our institutions and the many colleagues who helped us in different ways during this project for their comments and suggestions, especially to Professor Dr. Marcus Aguiar. EDB is grateful

406 for the Ph.D. degree scholarship provided by Capes (Coordenação de Aperfeiçoamento 407 de Pessoal de Nível Superior). EDB and SBLA acknowledge the computational support from Professor Carlos M. de Carvalho at LFTC-DFis-UFPR. 
FINANCIAL SUPPORT

This study was supported by Brazilian Coordination for the Improvement of

412 Higher Education Personnel (CAPES). DP was supported by the São Paulo Research

413 Foundation (FAPESP), grants \#2018/11187-8 and \#2016/01343-7 (ICTP-SAIFR).

414

415 CONFLICT OF INTEREST

416 The authors declare that they have no conflict of interests.

417

\section{DATA ACCESSIBILITY STATEMENT}

The model, phylogenies and interactions of all analysed cases are available at

https://github.com/elviradbastiani/host_switching_model.

\section{REFERENCES}

Agosta, S.J., \& Brooks, D.R. (2020). The major metaphors of evolution: Darwinism then and now

424 (Vol. 2). Springer Nature, Switzerland AG. pp. XVII - 273.

425 Agosta, S.J. \& Klemens, J.A. (2008). Ecological fitting by phenotypically flexible genotypes:

426 implications for species associations, community assembly and evolution. Ecology Letters, 11,

$427 \quad 1123-1134$.

428 Agosta, S.J., Janz, N. \& Brooks, D.R. (2010). How specialists can be generalists: resolving the"

429 parasite paradox" and implications for emerging infectious disease. Zoologia, 27, 151-162.

430 Aldous, D.J. (2001). Stochastic models and descriptive statistics for phylogenetic trees, from Yule 431 to today. Statistical Science, 16, 23-34.3

432 Araujo, S.B., Braga, M.P., Brooks, D.R., Agosta, S.J., Hoberg, E.P., von Hartenthal, F.W. \&

433 Boeger, W.A. (2015). Understanding host-switching by ecological fitting. PLoS One, 10, 
Badets, M., Whittington, I., Lalubin, F., Allienne, J.F., Maspimby, J.L., Bentz, S. \& Verneau, O.

436 (2011). Correlating early evolution of parasitic platyhelminths to Gondwana breakup. Systematic

437 Biology, 60, 762-781.

438 Baselga, A. (2010). Partitioning the turnover and nestedness components of beta diversity. Global 439 ecology and biogeography, 19, 134-143.

440 Baselga, A. (2013a). Separating the two components of abundance-based dissimilarity: balanced 441 changes in abundance vs. abundance gradients. Methods in Ecology and Evolution, 4, 552-557.

442 Baselga, A. (2013b). Multiple site dissimilarity quantifies compositional heterogeneity among

443 several sites, while average pairwise dissimilarity may be misleading. Ecography, 36, 124-128.

444 Baselga, A., Orme, D., Villeger, S., De Bortoli, J., Leprieur, F., Logez, M. \& Henriques-Silva, R.

445 (2017). Partitioning beta diversity into turnover and nestedness components. R package betapart, 446 Version, 1-4.

447 Bates, D., Maechler, M., Bolker, B., Walker, S., Christensen, R.H.B., Singmann, H. \& 448 Grothendieck, G. (2011). Package 'Ime4'. Linear mixed-effects models using S4 classes. R 449 package version, 1(6).

450 Becker, D.J., Streicker, D.G. \& Altizer, S. (2018). Using host species traits to understand the 451 consequences of resource provisioning for host-parasite interactions. Journal of Animal Ecology, $87,511-525$.

Blum, M.G. \& François, O. (2005). On statistical tests of phylogenetic tree imbalance: the Sackin and other indices revisited. Mathematical biosciences, 195, 141-153.

455 Boeger, W.A., Kritsky, D.C., Pie, M.R. \& Engers, K.B. (2005). Mode of transmission, host 456 switching, and escape from the Red Queen by viviparous gyrodactylids (Monogenoidea). Journal 457 of Parasitology, 91, 1000-1007. 
Host use dynamics in a heterogeneous fitness landscape generates oscillations in host range and

460 diversification. Evolution, 72, 1773-1783.

461 Braga, M.P. \& Janz, N. (2021). Host repertoires and changing insect-plant interactions.

462 Ecological Entomology, 46, 1241-1253.

463 Brooks, D.R. \& McLennan, D.A. (2012). The nature of diversity. University of Chicago Press, 464 pp. 676.

465 Brooks, D.R., Hoberg, E.P., Boeger, W.A., Gardner, S.L., Galbreath, K.E., Herczeg, D. \& 466 Dursahinhan, A.T. (2014). Finding them before they find us: informatics, parasites, and 467 environments in accelerating climate change. Comparative Parasitology, 81, 155-164.

468 Brooks, D.R., Hoberg, E.P. \& Boeger, W.A. (2019). The Stockholm paradigm: climate change 469 and emerging disease. University of Chicago Press, pp. 400.

470 Bush, A.O., Fernandez, J.C., Esch, G.W. \& Seed, J.R. (2001). Parasitism: the diversity and 471 ecology of animal parasites. Cambridge university press, pp. 540.

472 Bush, S.E., Sohn, E. \& Clayton, D.H. (2006). Ecomorphology of parasite attachment: experiments 473 with feather lice. Journal of Parasitology, 92, 25-31.

474 Charleston, M.A. \& Robertson, D.L. (2002). Preferential host switching by primate lentiviruses 475 can account for phylogenetic similarity with the primate phylogeny. Systematic biology, 51, 528535.

477 Costa, C.L., Lemos-Costa, P., Marquitti, F.M., Fernandes, L.D., Ramos, M.F., Schneider, D.M. 478 \& de Aguiar, M.A. (2019). Signatures of microevolutionary processes in phylogenetic patterns. 479 Systematic biology, 68, 131-144. 
Cuthill, J.H. \& Charleston, M.A. (2013). A simple model explains the dynamics of preferential

481 host switching among mammal RNA viruses. Evolution: International Journal of Organic

482 Evolution, 67, 980-990.

483 Damas, J., Hughes, G.M., Keough, K.C., Painter, C.A., Persky, N.S., Corbo, M. \& Lewin, H.A.

484 (2020). Broad host range of SARS-CoV-2 predicted by comparative and structural analysis of

485 ACE2 in vertebrates. Proceedings of the National Academy of Sciences, 117, 22311-22322.

486 De Vienne, D.M., Giraud, T. \& Shykoff, J.A. (2007). When can host shifts produce congruent 487 host and parasite phylogenies? A simulation approach. Journal of evolutionary biology, 20, 14284881438.

489 De Vienne, D.M., Refrégier, G., López-Villavicencio, M., Tellier, A., Hood, M.E. \& Giraud, T. 490 (2013). Cospeciation vs host-shift speciation: methods for testing, evidence from natural 491 associations and relation to coevolution. New Phytologist, 198, 347-385.

492 Doña, J., Sweet, A.D., Johnson, K.P., Serrano, D., Mironov, S. \& Jovani, R. (2017).

493 Cophylogenetic analyses reveal extensive host-shift speciation in a highly specialized and host-

494 specific symbiont system. Molecular phylogenetics and evolution, 115, 190-196.

495 Dowton, M. \& Austin, A.D. (1995). Increased genetic diversity in mitochondrial genes is 496 correlated with the evolution of parasitism in the Hymenoptera. Journal of Molecular Evolution, $497 \quad 41,958-965$.

498 Engelstädter, J. \& Fortuna, N.Z. (2019). The dynamics of preferential host switching: Host 499 phylogeny as a key predictor of parasite distribution. Evolution, 73, 1330-1340.

500 Engelstädter, J. \& Hurst, G.D. (2006). The dynamics of parasite incidence across host species. 501 Evolutionary Ecology, 20, 603-616.

502 Fecchio, A., Wells, K., Bell, J.A., Tkach, V.V., Lutz, H.L., Weckstein, J.D. \& Clark, N.J. (2019).

503 Climate variation influences host specificity in avian malaria parasites. Ecology Letters, 22, 547- 
505 Fox, J. \& Weisberg, S. (2019). Nonlinear Regression, Nonlinear Least Squares, and Nonlinear

506 Mixed Models in R. population, 150, 200.

507 Gavrilets, S. \& Hastings, A. (1996). Founder effect speciation: a theoretical reassessment. The

508 American Naturalist, 147, 466-491.

509 Hayward, A., Poulin, R. \& Nakagawa, S. (2021). A broadscale analysis of host-symbiont

510 cophylogeny reveals the drivers of phylogenetic congruence. Ecology Letters, 24, 1681-1696.

511 Higgs, P.G. \& Derrida, B. (1991). Stochastic models for species formation in evolving

512 populations. Journal of Physics A: Mathematical and General, 24, L985.

513 Imrie, R. M., Roberts, K. E. \& Longdon, B. (2021). Between virus correlations in the outcome of

514 infection across host species: Evidence of virus by host species interactions. Evolution letters, 5,

$515 \quad 472-483$.

516 Nylin, S. \& Janz, N. (2008). 15. The Oscillation Hypothesis Of Host-Plant Range And Speciation.

517 In Specialization, Speciation, and Radiation. University of California Press, 203-2015.

518 Jaramillo, M. \& Rivera-Parra, J. L. (2018). Host-Switching: How It Starts. In Disease Ecology.

519 Springer, Cham, pp. 139-156.

520 Kembel, S.W., Cowan, P.D., Helmus, M.R., Cornwell, W.K., Morlon, H., Ackerly, D.D. \& Webb,

521 C.O. (2010). Picante: R tools for integrating phylogenies and ecology. Bioinformatics, 26, 1463-

5221464.

523 Krasnov, B.R., Shenbrot, G.I., Khokhlova, I.S. \& Degen, A.A. (2016). Trait-based and

524 phylogenetic associations between parasites and their hosts: a case study with small mammals

525 and fleas in the Palearctic. Oikos, 125, 29-38.

526 Kuzmin, Y., de Vasconcelos Melo, F.T. \& Dos Santos, J.N. (2014). A new species of

527 Serpentirhabdias Tkach, Kuzmin \& Snyder, 2014 (Nematoda: Rhabdiasidae) parasitic in the 
528 brown ground snake Atractus major Boulenger (Reptilia: Serpentes: Dipsadidae) in Brazil.

529 Systematic Parasitology, 89, 101-106.

530 Light, J.E. \& Hafner, M.S. (2007). Cophylogeny and disparate rates of evolution in sympatric

531 lineages of chewing lice on pocket gophers. Molecular phylogenetics and evolution, 45, 997-

5321013.

533 Malcicka, M., Agosta, S.J. \& Harvey, J.A. (2015). Multi level ecological fitting: indirect life

534 cycles are not a barrier to host switching and invasion. Global change biology, 21, 3210-3218.

535 Manzo, F. \& Peliti, L. (1994). Geographic speciation in the Derrida-Higgs model of species

536 formation. Journal of Physics A: Mathematical and General, 27, 7079.

537 Mayr, E. (1999). Systematics and the origin of species, from the viewpoint of a zoologist. Harvard 538 University Press, pp. 372.

539 Müller, M.I., Morais, D.H., Costa-Silva, G.J., Aguiar, A., Avila, R.W. \& da Silva, R.J. (2018).

540 Diversity in the genus Rhabdias (Nematoda, Rhabdiasidae): Evidence for cryptic speciation.

541 Zoologica Scripta, 47, 595-607.

542 Nylin, S., Agosta, S., Bensch, S., Boeger, W.A., Braga, M.P., Brooks, D.R. \& Janz, N. (2018).

543 Embracing colonizations: a new paradigm for species association dynamics. Trends in ecology \& 544 evolution, 33, 4-14.

545 Nylin, S., Slove, J. \& Janz, N. (2014). Host plant utilization, host range oscillations and 546 diversification in nymphalid butterflies: a phylogenetic investigation. Evolution, 68, 105-124.

547 Oksanen, J., Blanchet, F.G., Kindt, R., Legendre, P., Minchin, P.R., O’hara, R.B. \& Oksanen, 548 M.J.(2013). Package ‘vegan’. Community ecology package, version, 2, 1-295.

549 Paradis, E. \& Schliep, K. (2019). ape 5.0: an environment for modern phylogenetics and 550 evolutionary analyses in R. Bioinformatics, 35, 526-528. 
551 Patella, L., Brooks, D.R. \& Boeger, W.A. (2017). Phylogeny and ecology illuminate the evolution

552 of associations under the Stockholm paradigm: Aglaiogyrodactylus spp. (Platyhelminthes,

553 Monogenoidea, Gyrodactylidae) and species of Loricariidae (Actinopterygii, Siluriformes). Vie

554 Et Milieu, 67, 91-102.

Team, R.C. (2020). R: A Language and Environment for Statistical Computing. R Foundation for

Statistical Computing, Vienna, Austria: Available at: https://www. R-project. org/. Last accessed

10 november 2021.

Reed, D.L., Light, J.E., Allen, J.M. \& Kirchman, J.J. (2007). Pair of lice lost or parasites regained: the evolutionary history of anthropoid primate lice. Bmc Biology, 5, 1-11.

560 Revell, L.J. (2012). phytools: an R package for phylogenetic comparative biology (and other

561 things). Methods in ecology and evolution, 3, 217-223.

562 Team, R. (2020). RStudio: Integrated Development for R. RStudio, PBC, Boston, MA. Available

563 at: https://www.rstudio.com/. Last accessed 10 november 2021.

564 Streicker, D.G., Turmelle, A.S., Vonhof, M.J., Kuzmin, I.V., McCracken, G.F. \& Rupprecht, C.E.

565 (2010). Host phylogeny constrains cross-species emergence and establishment of rabies virus in bats. Science, 329, 676-679.

567 Willkens, Y., Furtado, A.P., Dos Santos, J.N. \& de Vasconcelos Melo, F.T. (2021). Do host

568 habitat use and cospeciation matter in the evolution of Oswaldocruzia (Nematoda, Molineidae)

569 from neotropical amphibians? Journal of Helminthology, 95, e33.

570 Yule, U.G. (1925). A mathematical theory of evolution, based on the conclusions of Dr. JC Willis,

571 FRS. Philosophical Transactions of the Royal Society of London Series B, 213, 21-87. 
bioRxiv preprint doi: https://doi.org/10.1101/2021.11.27.470149; this version posted November 28, 2021. The copyright holder for this preprint (which was not certified by peer review) is the author/funder, who has granted bioRxiv a license to display the preprint in perpetuity. It is made available under aCC-BY-NC-ND 4.0 International license. 


\section{Tables}

Table 1. Description of the host sample size and parasite richness for each empirical study, of which host phylogenies were used as model parameters and host-parasite association to validate the simulations. All parasite groups are monoxenic, and the transmission in ectoparasites includes the larval and adult stages, and endoparasites' transmission includes only the larval. Legend: ID = Empirical study.

\begin{tabular}{|c|c|c|c|c|c|c|c|}
\hline ID & Host group & $\begin{array}{c}\text { Host } \\
\text { richness }\end{array}$ & $\begin{array}{c}\text { Parasites } \\
\text { group }\end{array}$ & $\begin{array}{l}\text { Parasite } \\
\text { richness }\end{array}$ & $\begin{array}{c}\text { Parasitism } \\
\text { type }\end{array}$ & $\begin{array}{c}\text { Spatial } \\
\text { scale }\end{array}$ & Reference \\
\hline 1 & Bird & 11 & $\begin{array}{l}\text { Feather mites } \\
\text { (Trouessartia spp.) }\end{array}$ & 11 & ectoparasites & regional & Donã et al. 2017 \\
\hline 2 & Mammals & 6 & $\begin{array}{c}\text { Lice } \\
\text { (Pediculus spp. } \\
\text { and Pthirus spp.) }\end{array}$ & 7 & ectoparasites & regional & Reed et al. 2007 \\
\hline 3 & Wildlife & 9 & Arthropods* & 8 & ectoparasites & regional & Becker et al. 2018 \\
\hline 4 & Rodents & 129 & Fleas* & 202 & ectoparasites & regional & Krasnov et al. 2016 \\
\hline 5 & Rodents & 11 & Fleas* & 19 & ectoparasites & local & Krasnov et al. 2016 \\
\hline 6 & Fish & 8 & $\begin{array}{l}\text { Platyhelminthes } \\
\text { (Gyrodactylidae) }\end{array}$ & 16 & ectoparasites & local & Patella et al. 2017 \\
\hline 7 & Frogs & 15 & $\begin{array}{c}\text { Polystomes } \\
\text { (Polystomatidae) }\end{array}$ & 13 & endoparasites & regional & Badets et al. 2011 \\
\hline 8 & Frogs & 31 & $\begin{array}{c}\text { Nematodes } \\
\text { (Rhabdias spp.) }\end{array}$ & 18 & endoparasites & regional & Müller et al. 2018 \\
\hline
\end{tabular}


bioRxiv preprint doi: https://doi.org/10.1101/2021.11.27.470149; this version posted November 28, 2021. The copyright holder for this preprint (which was not certified by peer review) is the author/funder, who has granted bioRxiv a license to display the preprint in perpetuity. It is made available under aCC-BY-NC-ND 4.0 International license.

$9 \quad$ Frogs $7 \quad$ Nematodes

5 endoparasites

local

Willkens et al. 2021

\section{(Oswaldocruzia}

spp.)

*include different parasite groups. 


\section{Figures}

a.

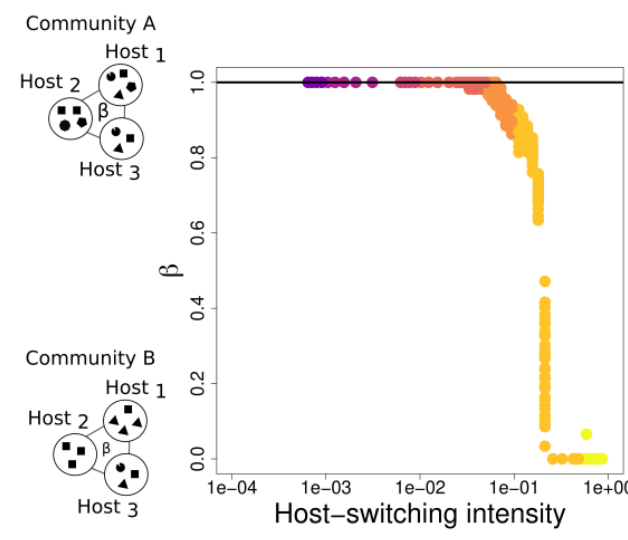

c.

b.

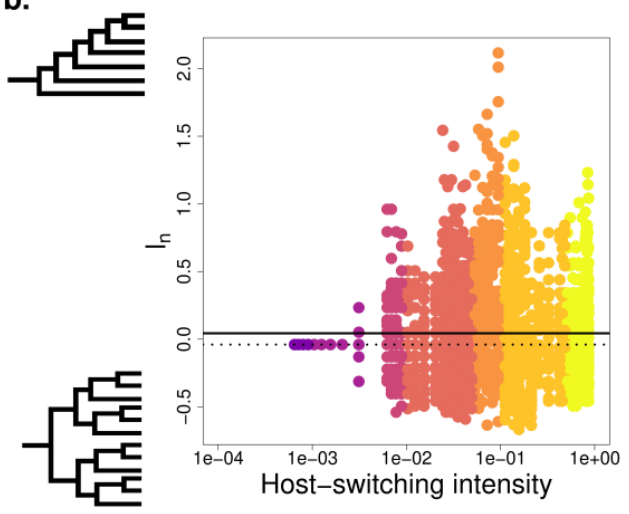

Host-switching intensity

$(50 \%, 100 \%]$

$(10 \%, 50 \%]$

$(5 \%, 10 \%]$

- $(1 \%, 5 \%]$

$(0.5 \%, 1 \%]$

$(0.1 \%, 0.5 \%]$

$(0.05 \%, 0.1 \%]$

$(0.01 \%, 0.05 \%]$

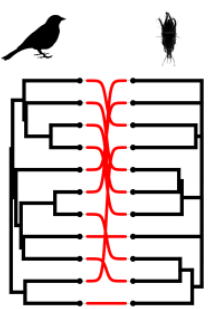

*Empiric data

Figure 1. Influence of host-switching on the eco-evolutionary patterns of simulated parasites for feather mites associated with birds (see Table 1 for details). Here we demonstrated the relationship between: a. Host-switching intensity and variation in the composition of parasite species $(\beta)$ between host species; $\mathbf{b}$. Host-switching intensity and parasite normalized Sackin index $\left(I_{n}\right)$; c. Relationship between $\beta, I_{n}$, and the host-switching intensity. The lines refer to empirical information of parasite (continuous) and host (dotted). The colored dots are redundant with the $\mathrm{x}$-axis scale of graphs (a) and (b), but intend to guide the interpretation of (c). A total of 50 runs were performed with 250 individuals of carrying capacity, for each configuration of the parameters of host-switching intensity. 

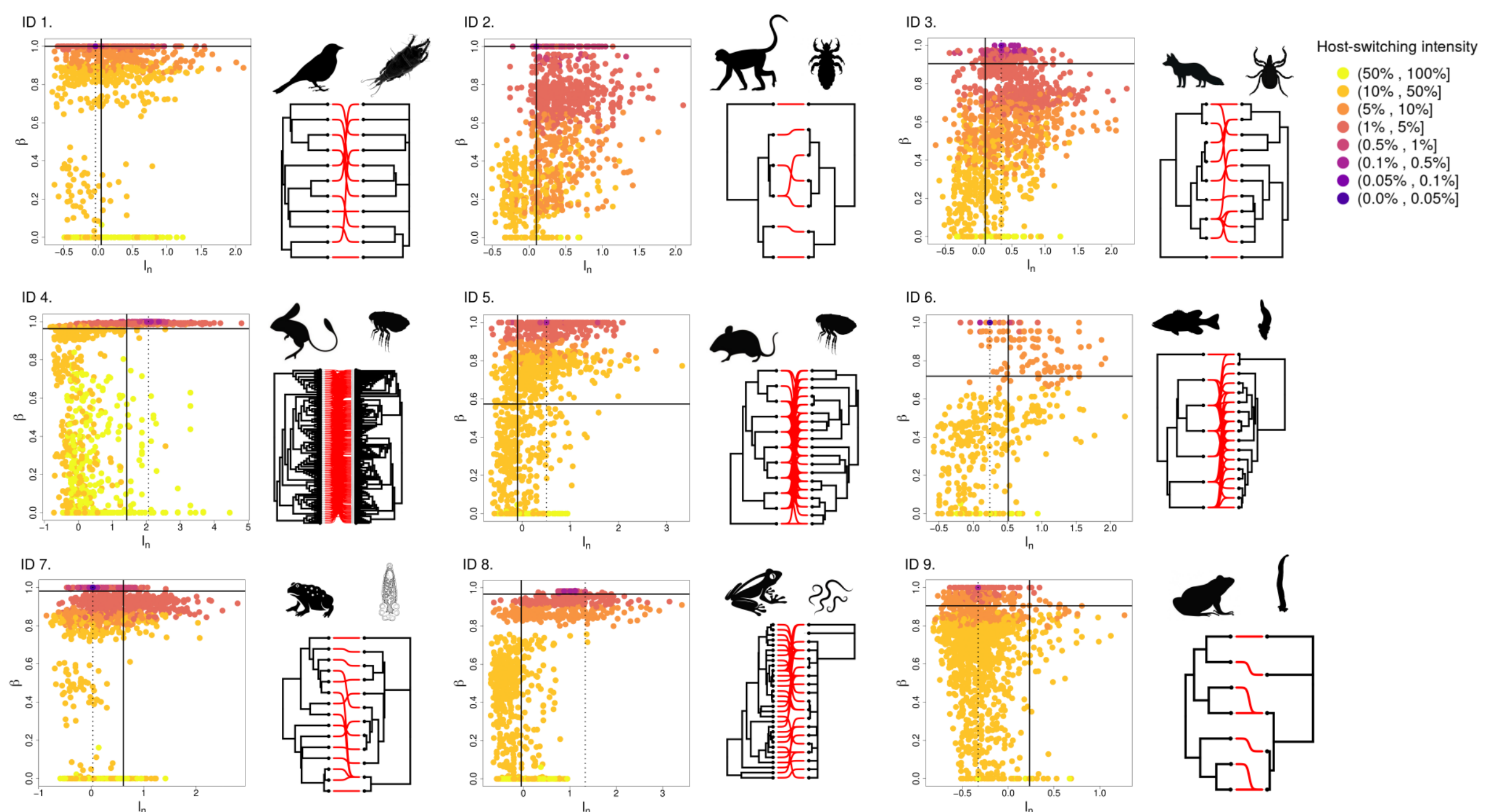

Figure 2. The relationship between variation in the composition, normalized Sackin index of parasite species and host-switching intensity for nine empirical cases: the variation in parasites' composition, measured by the metric beta diversity $(\beta)$ on the $y$-axis and the normalized Sackin index $\left(I_{n}\right)$ on the $x$-axis. Each ID represents an empirical case. The lines refer to empirical 
information of parasite (continuous) and host (dotted). Color scales represent each percentage interval of host-switching intensity. A total of 50 runs were performed with 250 individuals of carrying capacity, for each configuration of the parameters of host-switching intensity. 


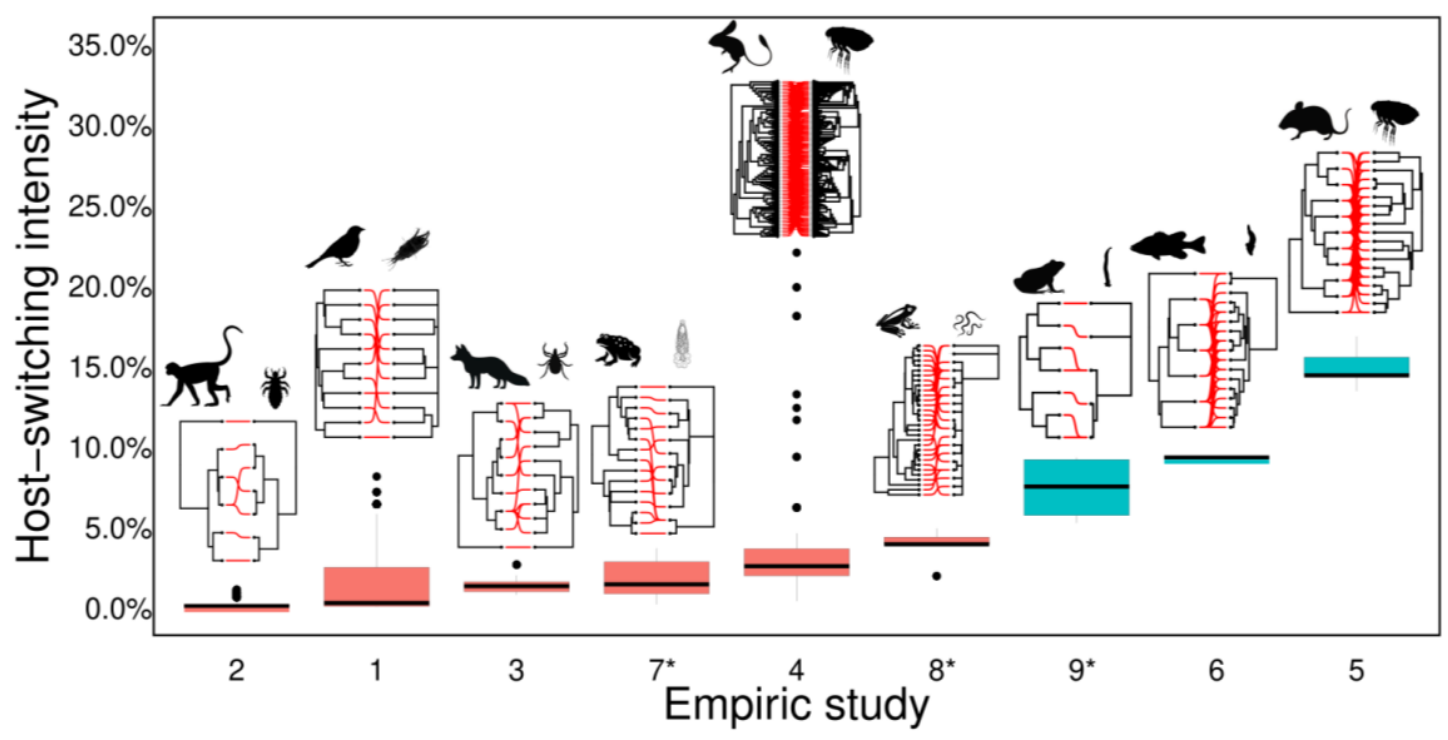

Figure 3. Relation of the host-switching intensity among empirical cases considering cases that correspond to $\pm 5 \%$ confidence interval in relation to the metrics variation (beta diversity and the normalized Sackin index simulated and empirical). The boxplots show the simulation distributions for each empirical case. Regional scale cases are represented by salmon color, and local scale cases by blue. The number in axis $\mathrm{x}$ represents the empirical cases: ID. 1 - Birds and feather mites. ID. 2 - Mammals and lice. ID. 3 - Wildlife and ectoparasites. ID. 4 and 5 - Rodents and fleas. ID. 6 - Fish and Platyhelminthes (Gyrodactylidae). ID. 7 - Frogs and Polystomes (Polystomatidae). ID. 8 - Frogs and Nematodes (Rhabdias spp.). ID. 9 - Frogs and Nematodes (Oswaldocruzia spp.). The asterisks (*) represent cases of endoparasites. 